\title{
Soliton wavespeed management: slowing, stopping, or reversing a solitary wave
}

\author{
Luke W. S. Baines and Robert A. Van Gorder* \\ Mathematical Institute, University of Oxford, Andrew Wiles Building, \\ Radcliffe Observatory Quarter, Woodstock Road, Oxford, OX2 6GG, UK
}

\begin{abstract}
While dispersion management is a well-known tool to control soliton properties such as shape or amplitude, far less effort has been directed toward the theoretical control of soliton wavespeed. However, recent experiments concerning the stopping or slowing of light demonstrate that the control of soliton wavespeed is of experimental interest. Motivated by these and other studies, we propose a management approach for modifying the wavespeed of a soliton (or of other nonlinear wave solutions, such as periodic cnoidal waves) under the nonlinear Schrödinger equation. Making use of this approach, we are able to slow, stop, or even reverse a solitary wave, and we give several examples to bright solitons, dark solitons, and periodic wavetrains, to demonstrate the method. An extension of the approach to spatially heterogeneous media, for which the wave may propagate differently at different spatial locations, is also discussed.
\end{abstract}

\section{INTRODUCTION}

The nonlinear Schrödinger (NLS) equation

$$
i \frac{\partial \Psi}{\partial t}+\frac{1}{2} \frac{\partial^{2} \Psi}{\partial x^{2}}+g|\Psi|^{2} \Psi=0,
$$

given here in dimensionless form, is known to admit solitary wave solutions, including solitons, and hence has attracted much attention in the literature. In addition to the solutions it naturally admits, there have been a variety of modifications to the structure of the NLS equation with the goal of modifying the emergent solitary wave solutions. One popular approach for modification of nonlinear waves in optics is dispersion management [1-3]. The approach involves temporal or spatial modification of the dispersion coefficient, and may also include damping and/or periodic amplification of the pulse. While more common in optics, dispersion management has also seen application in the modification of atomic matter waves [4] and Bose-Einstein condensates [5].

Soliton management has been used to modify properties such as the amplitude or width of a solitary wave $[6,7]$, with the more general combination of dispersion management, nonlinearity management, and loss/gain management resulting in a kind of self-similar transform of the original wave variables. Mathematically, the study of soliton management involves solving various general nonlinear Schrödinger equations having space and time varying coefficients (i.e. non-autonomous NLS equations); see, for instance, [8-17]. Such approaches have also recently been applied to non-autonomous problems involving parity-time (PT) symmetric potentials [18, 19].

Variational approaches have been used to approximate solution envelopes for solitary waves under various forms of soliton management $[3,20-22]$. The existence of the Lax representation for a model of soliton management under certain conditions was demonstrated in [23, 24]. In

* Robert.VanGorder@maths.ox.ac.uk addition to solitons or solitary waves, dispersion and nonlinearity management have also been applied to returnto-zero pulses [25]. Dispersion management not only allows for the control of wave properties, but can also be employed to stabilize such waves against perturbations [26]. Soliton management has also seen a good deal of application in periodic systems [27].

One possibility seemingly not explored in the literature is the use of an appropriate soliton management scheme in order to modify the propagation of a traveling wave. The results in [6] correspond to a modification of a standing wave, which the approach converting the particular shape or form of the wave via a kind of self-similar solution. Further, since that approach modifies the form of such a wave, the wave is may not be a true soliton in some cases (rather, it is more generally a solitary wave). However, motivated by the approach in [6] and related works, we consider a related approach aimed not at changing the soliton structure or envelope, but rather its wavespeed. To do so, we shall employ soliton management in such a way that a traveling solitary wave will have non-constant wavespeed (depending on time, or, in some cases, on both time and space), yet the solitary wave will maintain its form as it travels. Using such approach, one may alter the trajectory of a traveling solitary wave, slowing, stopping, or reversing it. When this wave is a soliton, it will remain a soliton, provided that the management is carried out in such a way that permits the wave to maintain its form both while propagating and after interaction with similar waves [28].

In the present paper, we shall explore the use of soliton management to modify the wavespeed of solitary traveling waves, including solitons. The approach is shown to slow, stop, or even reverse the propagation direction of the wave. In Sec. II we outline the general approach of soliton wavespeed management. In Sec. III, we apply soliton wavespeed management to slow, stop, or reverse a variety of solitary waves, including bright and dark soliton. Spatial heterogeneity can at times arise in physical systems, and in Sec. IV we discuss wavespeed management involving functions of space as well as time. We give concluding remarks in Sec. V. 


\section{SOLITON WAVESPEED MANAGEMENT}

Akin to what was done in [6], we consider the nonlinear Schrödinger equation for a homogeneous media,

$$
i \frac{\partial \Psi}{\partial t}+\frac{1}{2} D(t) \frac{\partial^{2} \Psi}{\partial x^{2}}+G(t)|\Psi|^{2} \Psi=0 .
$$

We assume a fairly generic solution of the form

$$
\Psi(x, t)=A(t) \exp (i[k x-\omega(t) t]) \phi(z),
$$

where the real-valued wave envelope $\phi$ depends on wave variable $z=x-c(t) t$, hence the wave moves with nonconstant wavespeed $c(t)$. Note that this solution is of a traveling wave form, rather than a self-similar form which is seemingly common in the dispersion management literature [6]. This point of departure from the literature will allow us to construct nonlinear waves which maintain their form as they propagate, rather than waves which deform in a self-similar manner as they move. The latter are no longer solitons, but rather solitary waves, as they do not maintain their form.

Placing the general solution (3) into (2), we obtain the two real-valued equations

$$
\begin{gathered}
\frac{d A}{d t} \phi+A\left(k D-\frac{d}{d t}(c t)\right) \frac{d \phi}{d z}=0, \\
\frac{A D}{2} \frac{d^{2} \phi}{d z^{2}}+A\left(\frac{d}{d t}(\omega t)-\frac{k^{2}}{2} D\right) \phi+A^{3} G \phi^{3}=0 .
\end{gathered}
$$

For a bounded wave envelope $\phi,(4)$ implies that $A(t)=$ $A_{0}$, a constant, while

$$
D(t)=\frac{1}{k} \frac{d}{d t}(c(t) t) .
$$

To continue further with (5), note that tanh or sech soliton solutions for $\phi$ will obey an ordinary differential equation of the form

$$
\frac{d^{2} \phi}{d z^{2}}+\alpha \phi+\beta \phi^{3}=0
$$

In particular, if $\alpha=-1, \beta=2$ then (7) permits the exact solution $\phi(z)=\operatorname{sech}(z)$ (a bright soliton), while if $\alpha=2, \beta=-2$ then (7) permits the exact solution $\phi(z)=\tanh (z)$ (a dark soliton). For other values of $\alpha$ and $\beta,(7)$ results in Jacobi elliptic solutions giving periodic rather than solitary waves. Therefore, to get (5) into the form of (7), we must have

$$
\begin{gathered}
\frac{\alpha A_{0}}{2} D(t)=A_{0}\left(\frac{d}{d t}(\omega(t) t)-\frac{k^{2}}{2} D(t)\right), \\
\frac{\beta A_{0}}{2} D(t)=A_{0}^{3} G(t) .
\end{gathered}
$$

The conditions (8)-(9) imply that

$$
\begin{gathered}
\omega(t)=\frac{k^{2}+\alpha}{2 k} c(t), \\
G(t)=\frac{\beta}{2 k A_{0}^{2}} \frac{d}{d t}(c(t) t) .
\end{gathered}
$$

Therefore, with $D(t)$ and $G(t)$ selected as in (6) and (11), respectively, we have the exact solution

$$
\Psi(x, t)=A_{0} \exp \left(i\left\{k x-\left(\frac{k^{2}+\alpha}{2 k}\right) c(t) t\right\}\right) \phi(z)
$$

to $(2)$, where $z=x-c(t) t$ and $\phi(z)$ satisfies $(7)$.

With this, we may choose $D(t)$ and $G(t)$ so that the wavespeed $c(t)$ obeys some desired properties. In particular, the approach may be used to slow, to stop, or to reverse the solitary wave. In what follows, we demonstrate this for bright and dark NLS solitons.

\section{SLOWING, STOPPING, OR REVERSING A NONLINEAR WAVE}

We shall first apply the general wavespeed management approach to a bright soliton solution, before giving corresponding conditions for the wavespeed management of dark solitons as well as periodic waves (snoidal, cnoidal, and dnoidal waves). Recall that this case corresponds to $\alpha=-1$ and $\beta=2$ in (7), which gives the exact NLS wavefunction solution $\phi(z)=\operatorname{sech}(z)$. The exact solution is then

$$
\Psi(x, t)=A_{0} \exp \left(i\left\{k x-\left(\frac{k^{2}-1}{2 k}\right) c(t) t\right\}\right) \operatorname{sech}(x-c(t) t)
$$

while the soliton intensity is given by

$$
|\Psi(x, t)|^{2}=A_{0}^{2} \operatorname{sech}^{2}(x-c(t) t)
$$

The peak of the wave is located at $x=X(t)$, where $X(t)=c(t) t$. The change in this position is then given by

$$
\frac{d X(t)}{d t}=\frac{d}{d t}(c(t) t)=\sigma(t)
$$

In order to control this position as a function of time (and hence control the wave velocity), we shall treat $\sigma(t)$ as a control function. This is equivalent to dispersion and nonlinearity management, as from (6) and (11) we have

$$
D(t)=\frac{1}{k} \sigma(t) \quad \text { and } \quad G(t)=\frac{1}{k A_{0}^{2}} \sigma(t) \text {. }
$$




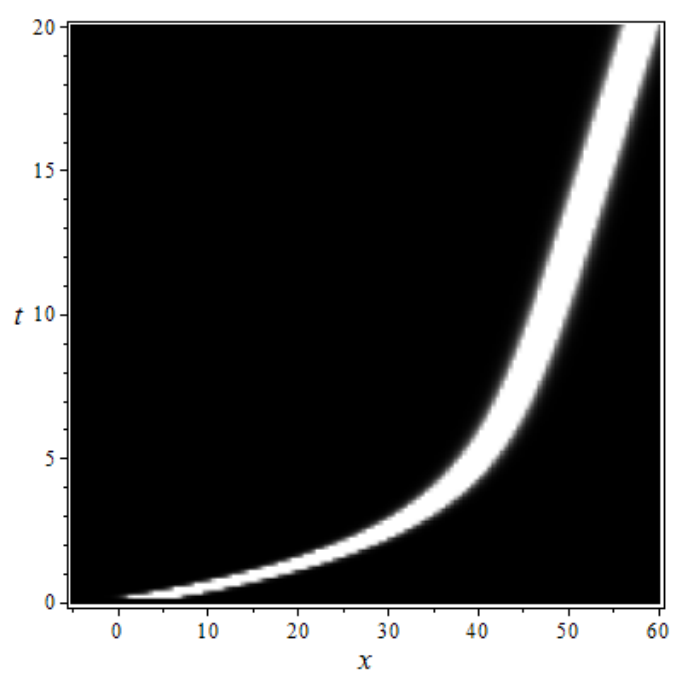

FIG. 1. Plot of $|\Psi|^{2}$ for a slowed bright soliton, using wavespeed management resulting in a wavespeed of the form (18). We choose $\mu=0.5, C_{0}=20, C_{\infty}=1$.

\section{A. Slowing a bright soliton}

In order to slow the bright soliton, we should have $\frac{d X}{d t}(0)>\frac{d X}{d t}(T)$, where $T>0$ is some terminal time. This is equivalent to choosing $c(t)$ so that

$$
c(0)>c(T)+T \frac{d c}{d t}(T) .
$$

The choice of $c(t)$ will enable us to modify the way in which this slowing is achieved.

For example, if we want to slow the wave gradually over time, then we may choose

$$
c(t)=C_{\infty}+\frac{C_{0}-C_{\infty}}{\mu}\left(\frac{1-\exp (-\mu t)}{t}\right),
$$

for $C_{0}>C_{\infty}>0$ and $\mu>0$ constants. In this example, we have that

$$
\sigma(t)=C_{\infty}+\left(C_{0}-C_{\infty}\right) \exp (-\mu t) .
$$

Then, $\frac{d X(t)}{d t}(0)=C_{0}$, while $\frac{d X(t)}{d t} \rightarrow C_{\infty}$ as $t \rightarrow \infty$. The parameter $\mu$ can be used to adjust the rate of the slowing, i.e. the deceleration of the soliton. For instance, $\frac{d^{2} X(t)}{d t^{2}}(0)=\left(C_{0}-C_{\infty}\right) \mu$. We plot one such solution in Figure 1 .

\section{B. Stopping a bright soliton}

In order to stop the bright soliton, we should have $\frac{d X}{d t}(T)=0$, where $T \in\left[T_{1}, T_{2}\right]$ is the time interval on which the soliton is stopped. One way to achieve this is

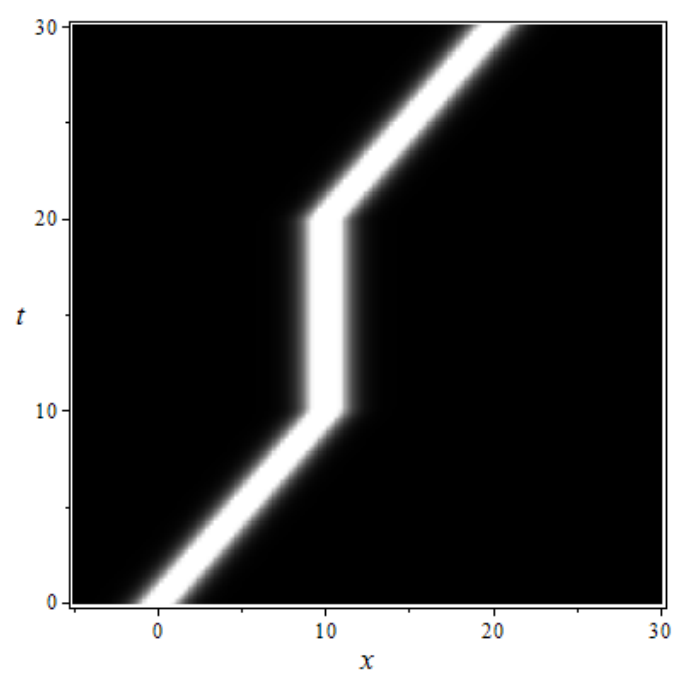

FIG. 2. Plot of $|\Psi|^{2}$ for a stopped bright soliton, using wavespeed management resulting in a wavespeed of the form (22). We choose $T_{1}=10$ and $T_{2}=20$, so that the soliton is stopped for 10 time units. We choose $C(\tau)=1$, so that the wavespeed is equal to unity when the soliton is not stopped.

to choose $\sigma(t)$ so that

$$
\sigma(t)= \begin{cases}0 & \text { if } t \in\left[T_{1}, T_{2}\right] \\ C_{0} & \text { otherwise }\end{cases}
$$

Integrating (15) in time, we find that $c(t) t=\sigma(t) t$ for this case, hence $c(t)=\sigma(t)$.

More generally, consider

$$
\sigma(t)= \begin{cases}0 & \text { if } t \in\left[T_{1}, T_{2}\right] \\ C(t) & \text { otherwise }\end{cases}
$$

where $C(t)$ is a positive, integrable function. This formulation permits variable wavespeeds when the soliton is not stopped. Integrating (15) in time, we find that

$$
c(t)= \begin{cases}0 & \text { if } t \in\left[T_{1}, T_{2}\right], \\ \frac{1}{t} \int_{0}^{t} C(\tau) d \tau & \text { otherwise }\end{cases}
$$

One such example is shown in Figure 2.

\section{Reversing a bright soliton}

In order to reverse the direction of the bright soliton, we should have $\frac{d X}{d t}$ change sign at some finite time, say $t=T>0$. To achieve this, we choose integrable $\sigma(t)$ such that $\sigma(t)>0$ for $t<T, \sigma(T)=0$, and $\sigma(t)<0$ for $t>T$.

As an example, choose

$$
\sigma(t)=C_{0} \tanh (T-t) .
$$




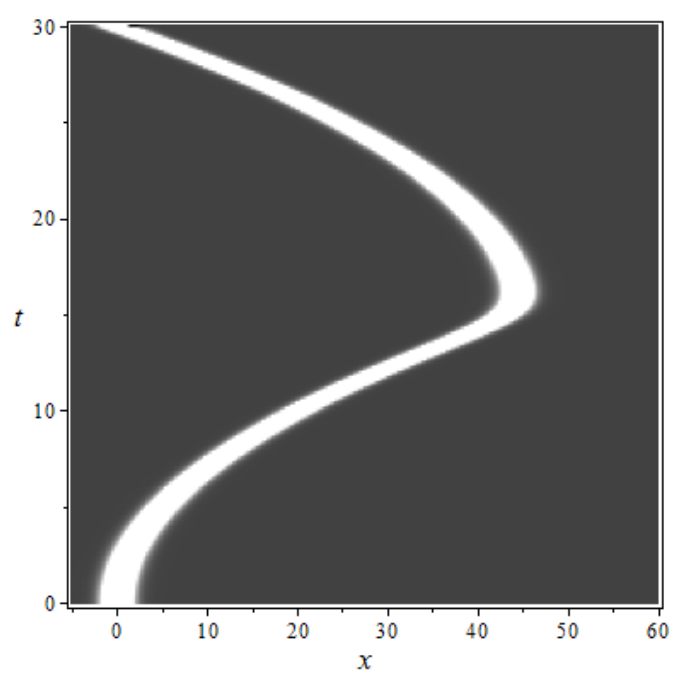

FIG. 3. Plot of $|\Psi|^{2}$ for a reversed bright soliton, using wavespeed management resulting in a wavespeed of the form (24). We choose $T=15$ so that the soliton is stopped at $t=15$ and then immediately reversed. We choose $C_{0}=0.2$.

Integrating (15) over time, we find

$$
c(t)=\frac{C_{0}}{t} \ln \left(\frac{\cosh (T)}{\cosh (T-t)}\right) .
$$

We plot one such example in Figure 3.

\section{Combination of management techniques}

It is possible to combine some of the above approaches in order to obtain other wavespeed controls. As an example, consider a situation where a wave is slowed for a time and later returned to a different wavespeed. To achieve this, we select

$$
\sigma(t)=J_{1} \tanh ^{2}(T-t)+J_{2} \tanh ^{3}(T-t),
$$

where

$$
J_{1}=J_{1}\left(C_{0}, C_{\infty}, T\right)=\frac{\left(C_{0}+C_{\infty} \tanh ^{3}(T)\right)}{(1+\tanh (T)) \tanh ^{2}(T)}
$$

and

$$
J_{2}=J_{2}\left(C_{0}, C_{\infty}, T\right)=\frac{\left(C_{0}-C_{\infty} \tanh ^{2}(T)\right)}{(1+\tanh (T)) \tanh ^{2}(T)}
$$

for constants $C_{0}, C_{\infty}, T>0$. This function has the properties that $\sigma(0)=C_{0}, \sigma(T)=0$, and $\sigma \rightarrow C_{\infty}$ as $t \rightarrow \infty$. Therefore, the position of the wave will slow from an initial wavespeed of $C_{0}$, briefly stop, and then resume propagation at a new wavespeed which tends asymptotically to $C_{\infty}$. Integrating (15) for $c(t)$, we obtain the

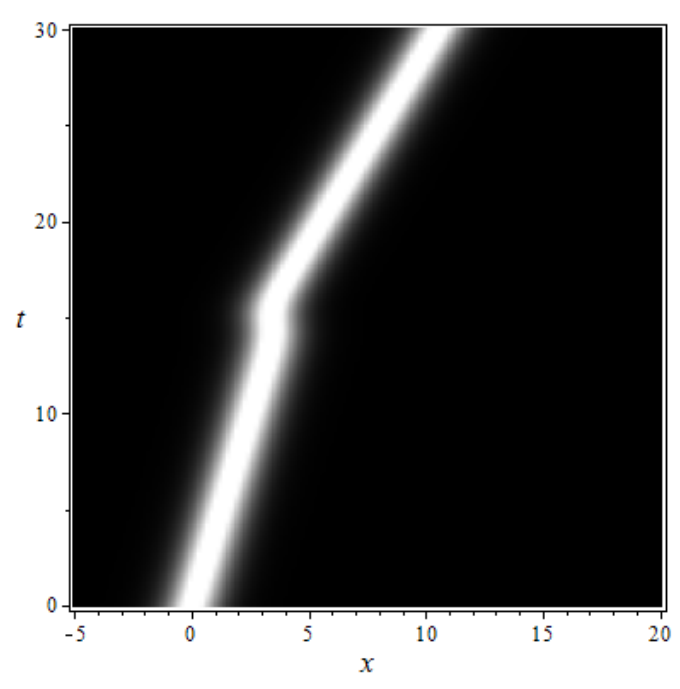

FIG. 4. Plot of $|\Psi|^{2}$ for a bright soliton using wavespeed management resulting in a wavespeed of the form (28). We choose $T=15, C_{0}=0.25$, and $C_{\infty}=0.5$, so that the wavespeed is doubled after $t=15$.

expression

$$
\begin{aligned}
c(t)= & J_{1}\left(1-\frac{\tanh (T)-\tanh (T-t)}{t}\right) \\
& -\frac{J_{2}}{2}\left(\frac{\tanh ^{2}(T)-\tanh ^{2}(T-t)}{t}\right) \\
& +\frac{J_{2}}{t} \ln \left(\frac{\cosh (T)}{\cosh (T-t)}\right) .
\end{aligned}
$$

We plot an example of such a solution in Figure 4 .

\section{E. Dark soliton management}

The same techniques we have employed for bright solitons can be applied to the management of dark solitons. To recover the dark soliton, we set $\alpha=2$ and $\beta=-2$ in (7), which gives the exact solution $\phi(z)=\tanh (z)$. The exact solution for the wave function to the NLS equation (2) is then

$\Psi(x, t)=A_{0} \exp \left(i\left\{k x-\left(\frac{k^{2}+2}{2 k}\right) c(t) t\right\}\right) \tanh (x-c(t) t)$

while the soliton intensity is given by

$$
|\Psi(x, t)|^{2}=A_{0}^{2} \tanh ^{2}(x-c(t) t) .
$$

The minimum of the soliton intensity (the center of the "dark" region) is located at $x=X(t)$, where $X(t)=$ $c(t) t$. The change in this position is again given by (15). For the dark soliton, the dispersion and nonlinearity management terms (6) and (11) become

$$
D(t)=\frac{1}{k} \sigma(t) \quad \text { and } \quad G(t)=-\frac{1}{k A_{0}^{2}} \sigma(t),
$$




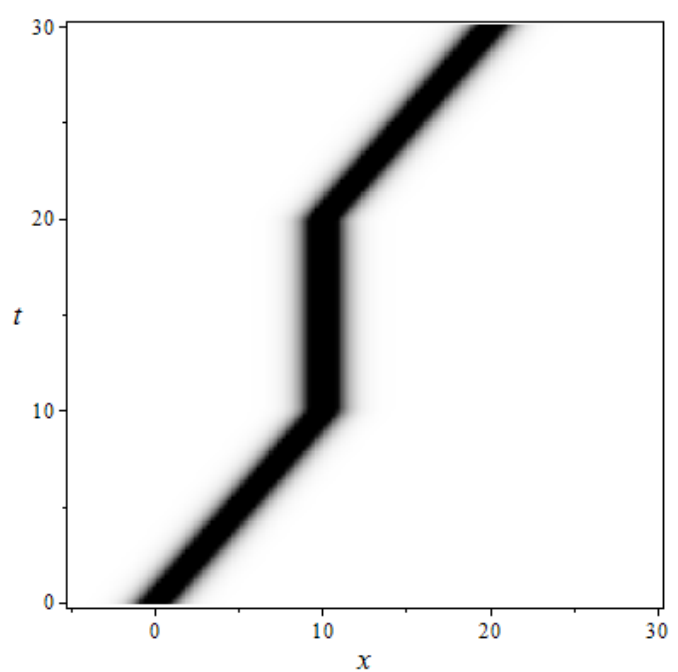

FIG. 5. Plot of $|\Psi|^{2}$ for a stopped dark soliton, using wavespeed management resulting in a wavespeed of the form (22). We choose $T_{1}=10$ and $T_{2}=20$, so that the soliton is stopped for 10 time units. We choose $C(\tau)=1$, so that the wavespeed is equal to unity when the soliton is not stopped.

respectively. Therefore, it is again sufficient to change $\sigma(t)$ in order to employ dispersion and nonlinearity management in a manner which will modify the wavespeed of the dark soliton. In Figure 5 we plot a dark soliton under the wavespeed management technique given in (22).

\section{F. Periodic wave management}

In addition to the bright and dark solitons discussed above, there are a variety of other nonlinear waves emergent from the NLS equation. Periodic nonlinear waves consisting of Jacobi elliptic functions sn, cn, and dn (snoidal, cnoidal, and dnoidal waves, respectively) have attracted interest [29-31], with dispersion management having been applied in [32]. We shall show here that the soliton wavespeed management approach can be applied to these nonlinear waves, as well.

In order to obtain the snoidal wave envelope $\phi(z)=$ $\operatorname{sn}(z, \ell), 0<\ell<1$, note that one requires

$$
\frac{d^{2} \phi}{d z^{2}}+\left(1+\ell^{2}\right) \phi-2 \ell^{2} \phi^{3}=0 .
$$

Comparing this with (7), we arrive at $\alpha=1+\ell^{2}, \beta=$ $-2 \ell^{2}$. The wavespeed managed wavefunction then reads

$\Psi(x, t)=A_{0} \exp \left(i\left\{k x-\left(\frac{k^{2}+\ell^{2}+1}{2 k}\right) c(t) t\right\}\right) \operatorname{sn}(z, \ell)$

with management terms

$$
D(t)=\frac{1}{k} \sigma(t) \quad \text { and } \quad G(t)=-\frac{\ell^{2}}{k A_{0}^{2}} \sigma(t) .
$$

In order to obtain the cnoidal wave envelope $\phi(z)=$ $\operatorname{cn}(z, \ell), 0<\ell<1$, note that one requires

$$
\frac{d^{2} \phi}{d z^{2}}+\left(1-2 \ell^{2}\right) \phi+2 \ell^{2} \phi^{3}=0 .
$$

Comparing this with (7), we arrive at $\alpha=1-2 \ell^{2}, \beta=$ $2 \ell^{2}$. The wavespeed managed wavefunction then reads

$$
\Psi(x, t)=A_{0} \exp \left(i\left\{k x-\left(\frac{k^{2}-2 \ell^{2}+1}{2 k}\right) c(t) t\right\}\right) \operatorname{cn}(z, \ell)
$$

with management terms

$$
D(t)=\frac{1}{k} \sigma(t) \quad \text { and } \quad G(t)=\frac{\ell^{2}}{k A_{0}^{2}} \sigma(t) .
$$

In order to obtain the dnoidal wave envelope $\phi(z)=$ $\operatorname{dn}(z, \ell), 0<\ell<1$, note that one requires

$$
\frac{d^{2} \phi}{d z^{2}}-\left(2-\ell^{2}\right) \phi+2 \phi^{3}=0 .
$$

Comparing this with (7), we arrive at $\alpha=-\left(2-\ell^{2}\right)$, $\beta=2$. The wavespeed managed wavefunction then reads

$$
\Psi(x, t)=A_{0} \exp \left(i\left\{k x-\left(\frac{k^{2}+\ell^{2}-2}{2 k}\right) c(t) t\right\}\right) \operatorname{dn}(z, \ell)
$$

with management terms

$$
D(t)=\frac{1}{k} \sigma(t) \quad \text { and } \quad G(t)=\frac{1}{k A_{0}^{2}} \sigma(t) .
$$

In Figure 6 we plot a cnoidal under the wavespeed management technique given in (22). Other periodic solutions and management techniques can similarly be considered, with analogous results.

\section{SPATIALLY HETEROGENEOUS MEDIA}

We now turn our attention to the NLS equation having space-varying coefficients [12, 33-35] (for wave propagation in a heterogeneous media), or with spatial heterogeneity corresponding to an applied field or confining potential (such as those found in various studies of spatially heterogeneous BECs [36-39] or in nonlinear optics [40-44]). Control of the quasi-soliton velocity by the use of spatiotemporal inhomogeneity has considered for a variety of equations in recent works on generalized nonlinear Schrödinger equations [45-50]. One such application is to situations where an optical fiber is spatially heterogeneous [47, 49].

Due to a generic lack of integrability, this case is naturally harder to study. However, we will arrive at conditions on the spatially heterogeneous wavespeed for which the wavespeed management approach can be carried out. 


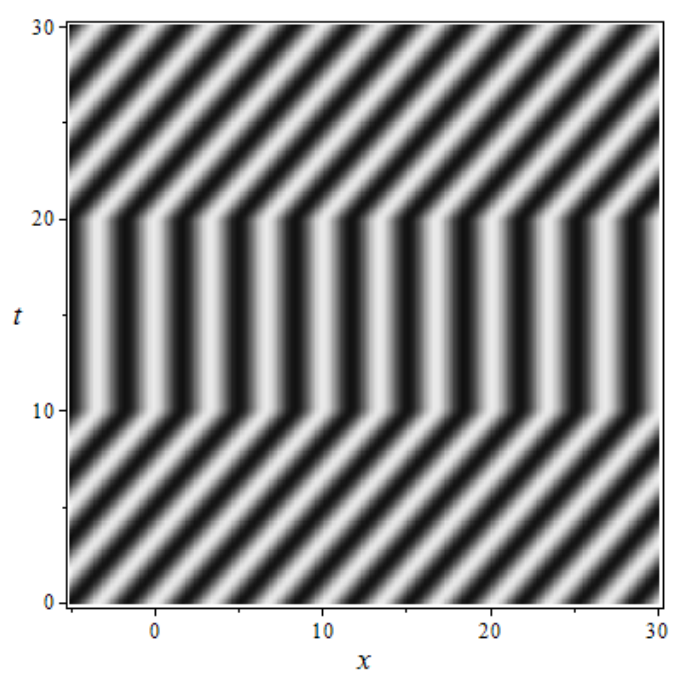

FIG. 6. Plot of $|\Psi|^{2}$ for a stopped cnoidal wave (with parameter $\ell=0.5$ ), using wavespeed management resulting in a wavespeed of the form (22). We choose $T_{1}=10$ and $T_{2}=20$, so that the solitary wave is stopped for 10 time units. We choose $C(\tau)=1$, so that the wavespeed is equal to unity when the soliton is not stopped.

With heterogeneous dispersion and nonlinear coefficients, we have

$$
i \frac{\partial \Psi}{\partial t}+\frac{1}{2} D(x, t) \frac{\partial^{2} \Psi}{\partial x^{2}}+G(x, t)|\Psi|^{2} \Psi=0 .
$$

We assume a more general form of the solution (3), which takes the form

$$
\Psi(x, t)=A(x, t) \exp (i[k x-\Omega(x, t)]) \Phi(Z),
$$

where the real-valued wave envelope $\Phi$ depends on wave variable $Z=x-C(x, t)$, hence the wave moves with nonconstant wavespeed $C(x, t)$. Assuming that $\Phi$ satisfies an ODE of the form

$$
\frac{d^{2} \Phi}{d Z^{2}}+\alpha \Phi+\beta \Phi^{3}=0
$$

we find that (43) is possible provided five conditions hold:

$$
\begin{gathered}
2 A_{x}\left(1-C_{x}\right)-A C_{x x}=0, \\
A_{t}+\frac{D}{2}\left(2 A_{x}\left(k-\Omega_{x}\right)-A \Omega_{x x}\right)=0, \\
-C_{t}+D\left(k-\Omega_{x}\right)\left(1-C_{x}\right)=0, \\
D=\frac{2 A \Omega_{t}}{A\left(\left(k-\Omega_{x}\right)^{2}+\alpha\left(1-C_{x}\right)^{2}\right)-A_{x x}}, \\
G=\frac{\beta}{2} \frac{D}{A^{2}}\left(1-C_{x}\right)^{2},
\end{gathered}
$$

where for ease of notation subscripts denote partial derivatives. The first three conditions remove secular growth of terms obtained when placing (42) in (41), while the final two arise from the restriction that (43) holds. Note that (47) and (48) determine the spatially heterogeneous management functions $D$ and $G$. Using (44), we must have

$$
A=\frac{a(t)}{\sqrt{1-C_{x}}} .
$$

From here, the remaining conditions are restrictions on $\Omega$ and $C$.

For the case of spatially homogeneous wavespeeds, $C(x, t)=c(t) t$, and condition (44) is satisfied, leaving $c(t)$ arbitrary and $a(t), \Omega$ to be determined by (45)-(46). However, in the spatially heterogeneous case, we must satisfy all three conditions, meaning that we are no longer free to choose $C(x, t)$ arbitrarily. Rather, the additional restriction on $C(x, t)$ ensures the integrability of the specific case we consider, as such integrability is implicit in the assumption of a traveling wave envelope governed by (43).

\section{A. An example of linear spatial heterogeneity}

While giving examples of $C(x, t)$ such that the needed conditions hold is challenging and will depend greatly on the specific application at hand, we can construct a relatively simple example to demonstrate the approach.

Consider $C(x, t)=(m-n x) f(t)$, for $m, n>0$ constant parameters and $f(t)$ a differentiable function of time. We find that $A(x, t)=A_{0}$ (a constant) and $\Omega(x, t)=p(m-n x) f(t)$ satisfy the conditions (44)-(45). Substituting (47) into (46), we find that (46) is satisfied provided $p=k=\sqrt{\alpha}$, and we therefore must have $\alpha>0$ for this family of solutions. This rules out the bright soliton, but dark solitons are still possible. We therefore have the wavefunction

$$
\Psi(x, t)=A_{0} \exp (i \sqrt{\alpha}[x-(m-n x) f(t)]) \Phi(Z),
$$

with $Z=x-(m-n x) f(t)$, provided $\Phi(Z)$ satisfies (43) for some $\alpha>0$ and the management terms are selected like

$$
\begin{gathered}
D=\frac{(m-n x) f^{\prime}(t)}{\sqrt{\alpha}(1+n f(t))^{2}}, \\
G=\frac{\beta}{2 \sqrt{\alpha} A_{0}^{2}}(m-n x) f^{\prime}(t) .
\end{gathered}
$$

Choosing $f(t)=g(t) t$, from $Z=x-C(x, t)=x-$ $(m-n x) g(t) t$, we have an effective wavespeed

$$
c(t)=\frac{m g(t)}{1+n g(t) t} .
$$




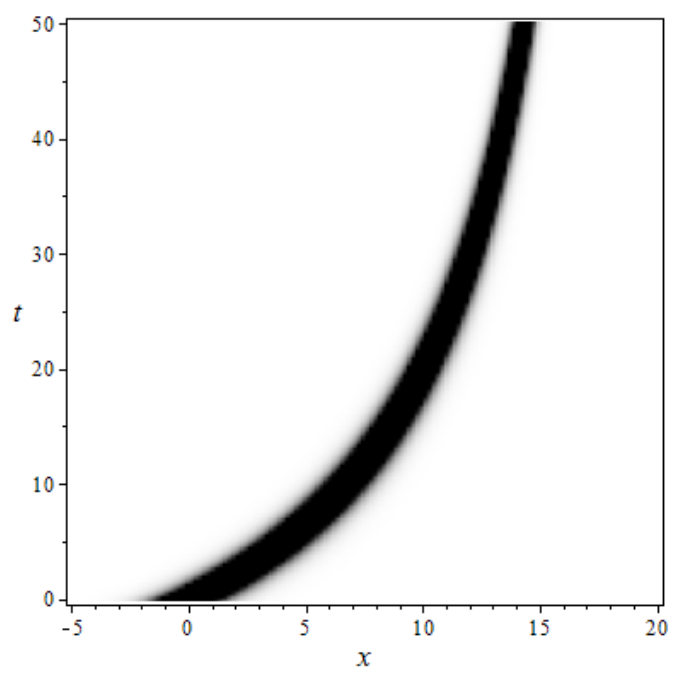

FIG. 7. Plot of $|\Psi|^{2}$ for a dark soliton (corresponding to $\alpha=$ $2, \beta=-2$ in (43)) using wavespeed management resulting in a spatially heterogeneous wave variable of the form $Z=$ $x-C(x, t)$ for $C(x, t)=(m+n x) t$. We choose $m=1$ and $n=$ -0.05 . Notice that the wave both slows (due to the decreasing effective wavespeed predicted in (53)) and is confined (due to the heterogeneity in the space variable).

This suggests that the wave should show as time increases, and asymptotically stop as $t \rightarrow \infty$. We plot one such example (for a dark soliton) in Figure 7, and indeed we find that the wave will gradually slow as time increases. Furthermore, due to the spatial heterogeneity of the wave variable $Z$, we observe that the wave envelope narrows as it propagates, rather than maintaining its form.

\section{CONCLUSIONS}

Motivated by experiments which slow or stop the motion of light (or other waves), we modify the approach of dispersion and nonlinearity management for the NLS equation to obtain a kind of wavespeed management. Through appropriate modification of the dispersion and nonlinearity coefficients, we are able to control the mo- tion of traveling solitons (and, more generally, nonlinear traveling waves). For appropriate choices of these coefficients, we may slow, stop, or even reverse the wave. Combinations of these approaches can be used to shift the wavespeed from one value to another, with still more complicated modifications possible. One may also choose spatially varying dispersion and nonlinearity coefficients, in order to modify the propagation of the wave in such a way that the wavespeed may vary locally over space in addition to time. The latter is a kind of hybrid approach to our wavespeed management and the spatial dispersion management of $[1-3,6,7]$.

We left much of the wavespeed management in the spatially heterogeneous regime unexplored, as this will be more analytically involved, and the form of the solitary wave (when it exists) will strongly depend on the particular form of the spatial heterogeneity employed (and hence on the particulars of the physical phenomena and experimental setup being considered). A more detailed consideration of such wavespeed management, particularly for NLS with spatial potential (as often arises in the study of spatially confined BECs) would be one useful direction for future work.

Orbital stability or instability of nonlinear waves is a useful indicator of whether waves will persist under small spectral perturbations [51-54]. In turn, such results are useful for understanding if a wave should persist in real experiments, since minor errors or spatial irregularities in an experiment may result in the loss of an intrinsically unstable wave. It may be possible to use the management approach discussed in Sec. IV to reinforce the wave envelope, making it more robust against spectral perturbations, and this would be another useful possibility for future work. Stabilization via wavespeed modification might prove useful for experimentally maintaining waves which are otherwise theoretically unstable (and are unlikely to be observed in experiments under normal conditions in the absence of such management). Soliton interaction under standard dispersion management was discussed in [55], with suppression of interaction forces between the dispersion managed solitons one of the focus points of that study. Therefore, it may be useful to study solitary wave interaction under the approach we propose; indeed, one may be able to use our approach to reinforce or stabilize solutions which might otherwise lose structure under standard dispersion management.
[1] T. Lakoba, J. Yang, D. Kaup, and B. Malomed, Optics communications 149, 366 (1998).

[2] M. J. Ablowitz and G. Biondini, Optics Letters 23, 1668 (1998).

[3] A. Biswas, Journal of Optics A: Pure and Applied Optics 4, 84 (2001).

[4] B. Eiermann, P. Treutlein, T. Anker, M. Albiez, M. Taglieber, K.-P. Marzlin, and M. Oberthaler, Physical review letters 91, 060402 (2003).
[5] P. Kevrekidis, G. Theocharis, D. Frantzeskakis, and B. A. Malomed, Physical review letters 90, 230401 (2003).

[6] V. Serkin and A. Hasegawa, Journal of Experimental and Theoretical Physics Letters 72, 89 (2000).

[7] V. N. Serkin and A. Hasegawa, IEEE Journal of selected topics in Quantum Electronics 8, 418 (2002).

[8] R. Hao, L. Li, Z. Li, W. Xue, and G. Zhou, Optics Communications 236, 79 (2004). 
[9] L. Wang, L. Li, Z. Li, G. Zhou, and D. Mihalache, Physical Review E 72, 036614 (2005).

[10] R. Hao, L. Li, R. Yang, Z. Li, and G. Zhou, Chinese Optics Letters 3, 136 (2005).

[11] R. Yang, L. Li, R. Hao, Z. Li, and G. Zhou, Physical Review E 71, 036616 (2005).

[12] V. Serkin, A. Hasegawa, and T. Belyaeva, Physical Review Letters 98, 074102 (2007).

[13] H.-G. Luo, D. Zhao, and X.-G. He, Physical Review A 79, 063802 (2009).

[14] X.-G. He, D. Zhao, L. Li, and H.-G. Luo, Physical Review E 79, 056610 (2009).

[15] Z.-Y. Yang, L.-C. Zhao, T. Zhang, Y.-H. Li, and R.-H. Yue, Optics Communications 283, 3768 (2010).

[16] Z.-Y. Yang, L.-C. Zhao, T. Zhang, Y.-H. Li, and R.-H. Yue, Physical Review A 81, 043826 (2010).

[17] F. Yu, Physical Review E 91, 032914 (2015).

[18] C.-Q. Dai and Y.-Y. Wang, Optics Communications 315, 303 (2014).

[19] M.-Z. Jin and J.-F. Zhang, Nonlinear Dynamics 87, 2179 (2017).

[20] S. K. Turitsyn and E. G. Shapiro, Optical Fiber Technology 4, 151 (1998).

[21] V. N. Serkin and A. Hasegawa, Physical Review Letters 85, 4502 (2000).

[22] A. Biswas, Chaos, solitons \& fractals 14, 447 (2002).

[23] V. N. Serkin and T. L. Belyaeva, Quantum Electronics 31, 1007 (2001).

[24] V. N. Serkin and T. L. Belyaeva, JETP Letters 74, 573 (2001).

[25] G. Biondini and S. Chakravarty, Optics letters 26, 1761 (2001).

[26] V. Zharnitsky, E. Grenier, C. K. Jones, and S. K. Turitsyn, Physica D: Nonlinear Phenomena 152, 794 (2001).

[27] B. A. Malomed, Soliton management in periodic systems (Springer Science \& Business Media, 2006).

[28] P. G. Drazin and R. S. Johnson, Solitons: an introduction, Vol. 2 (Cambridge university press, 1989).

[29] H. Shin, Physical Review E 63, 026606 (2001).

[30] J. C. Bronski, L. D. Carr, B. Deconinck, and J. N. Kutz, Physical Review Letters 86, 1402 (2001).

[31] J. C. Bronski, L. D. Carr, R. Carretero-González, B. Deconinck, J. N. Kutz, and K. Promislow, Physical Review E 64, 056615 (2001).

[32] Y. V. Kartashov, V. A. Vysloukh, E. Marti-Panameno, D. Artigas, and L. Torner, Physical Review E 68, 026613 (2003).

[33] T. Xu, C.-Y. Zhang, G.-M. Wei, J. Li, X.-H. Meng, and B. Tian, The European Physical Journal B 55, 323 (2007).

[34] J. Belmonte-Beitia and J. Cuevas, Journal of Physics A: Mathematical and Theoretical 42, 165201 (2009).

[35] C. Dai, Y. Wang, and C. Yan, Optics Communications 283, 1489 (2010).

[36] Z. Yan and C. Hang, Physical Review A 80, 063626 (2009).

[37] K. Mallory and R. A. Van Gorder, Physical Review E 90, 023201 (2014).

[38] K. Mallory and R. A. Van Gorder, Physical Review E 92, 013201 (2015).

[39] E. K. Luckins and R. A. Van Gorder, Annals of Physics 388, 206 (2018).

[40] F. K. Abdullaev, Wiley Series in Nonlinear Science, Chichester: Wiley,- c1994 (1994).

[41] L. Li, Z. Li, S. Li, and G. Zhou, Optics Communications 234, 169 (2004).

[42] T. Xu, J. Li, H.-Q. Zhang, Y.-X. Zhang, W. Hu, Y.-T. Gao, and B. Tian, Physics Letters A 372, 1990 (2008).

[43] L. Wu, J.-F. Zhang, L. Li, C. Finot, K. Porsezian, et al., Physical Review A 78, 053807 (2008).

[44] A. Choudhuri, H. Triki, and K. Porsezian, Physical Review A 94, 063814 (2016).

[45] X.-F. Zhang, L. Wen, X.-B. Luo, and Z.-W. Xie, Physics Letters A 376, 465 (2012).

[46] E. Kengne, Chaos, Solitons \& Fractals 61, 56 (2014).

[47] Y.-J. Feng, Y.-T. Gao, Z.-Y. Sun, D.-W. Zuo, Y.-J. Shen, Y.-H. Sun, L. Xue, and X. Yu, Physica Scripta 90, 045201 (2015).

[48] A. Arnaudon, Journal of Physics A: Mathematical and Theoretical 49, 125202 (2016).

[49] W. Liu, L. Huang, P. Huang, Y. Li, and M. Lei, Applied Mathematics Letters 61, 80 (2016).

[50] J.-J. Su, Y.-T. Gao, and S.-L. Jia, Communications in Nonlinear Science and Numerical Simulation 50, 128 (2017).

[51] T. Gallay and M. Hrgus, Journal of Dynamics and Differential Equations 19, 825 (2007).

[52] J. Bellazzini and N. Visciglia, Indiana University Mathematics Journal , 1211 (2010).

[53] R. A. Van Gorder, Journal of the Physical Society of Japan 83, 054005 (2014).

[54] T. Gallay and D. Pelinovsky, Journal of Differential Equations 258, 3607 (2015).

[55] R. Ganapathy, K. Porsezian, A. Hasegawa, and V. Serkin, IEEE journal of quantum electronics 44, 383 (2008). 